Ann. Biol. anim. Bioch. Biophys., I962, 2 (4), 36r-364.

\title{
ENREGISTREMENT DE L'ACTIVITÉ UNITAIRE DES AFFÉRENCES VAGALES. RÉCEPTION PAR MICROÉLECTRODES AU NIVEAU DU GANGLION PLEXIFORME
}

\author{
N. MEI \\ Laboratoire de Physiologie générale, Faculté des Sciences, Marseille \\ (Directeur : $M$. Dussardier)
}

\section{SOMMAIRE}

L'activité unitaire des neurones sensitifs du vague a été recueillie au niveau du ganglion plexiforme du Chat à l'aide de microélectrodes extracellulaires. Sur 20 animaux, nous avons pu étudier I30 unités. La plupart d'entre elles étaient d'origine respiratoire, mais nous avons trouvé également des unités d'origine cardiaque, œesophagienne ou gastrique. La technique utilisée semble pouvoir devenir une technique de routine pour l'étude des afférences végétatives.

La technique dite de la "fibre isolée " a permis un développement considérable de nos connaissances relatives à la physiologie des fibres sensitives. C'est en particulier grâce à son emploi que divers types de récepteurs ont pu être mis en évidence au niveau du cœur, des poumons, de l'estomac, etc. (Citons entre autres Adrian, I933 ; WhitTERIDGE, I948 ; PAINTAL, I952, I953, I954 ; IGGo, I955, I957, I958.) Nous nous sommes cependant demandé si l'enregistrement par microélectrodes de l'activité des cellules du ganglion plexiforme ne pourrait pas constituer, à bien des points de vue, une technique plus avantageuse pour l'étude des afférences vagales. L'exposé des résultats obtenus fait l'objet de cette note préliminaire.

\section{TECHNIQUES}

Nos expériences portent sur 20 chats d'un poids variant de I à $4 \mathrm{~kg}$. L'animal, préalablement anesthésié au Nembutal, est fixé sur le dos dans un appareil de contention. Une incision longitudinale médiane faite au niveau laryngé, suivie de l'ablation de la glande parotide, donne accès au ganglion plexiforme. La dissection de la capsule conjonctive du ganglion est effectuée soigneusement sous une loupe binoculaire. Nous écartons la carotide a fin de diminuer les mouvements parasites. Les électrodes utilisées sont constituées par des micropipettes de verre étirées à la main et remplies de $\mathrm{KCl}$ à concentration $3 \mathrm{M}$. 


\section{RÉSULTATS}

La réception d'activités unitaires s'est révélée facile au niveau du ganglion plexiforme. Lorsque nous en obtenons une il est généralement possible de la suivre pendant plus d'une heure. Nous avons ainsi enregistré l'activité de I30 neurones sensitifs. Les caractéristiques temporelles de leur décharge ou leur mode d'activation nous ont permis de reconnaître 4 groupes d'unités : 68 respiratoires, 9 cardiaques, I5 oesophagiennes et 4 gastriques. Les organes innervés par les autres neurones n'ont pas été précisés, en général pour des raisons purement instrumentales.

\section{Io) Unités respiratoires.}

Ce sont celles que nous avons obtenues le plus souvent. Quarante et une d'entre elles pulsaient uniquement à l'inspiration; la latence de leur réponse par rapport au début de l'inspiration était variable de 1'une à l'autre. Vingt-trois étaient continuellement actives et présentaient une augmentation de fréquence pendant l'inspiration. Enfin, chez un chat sous respiration artificielle, nous avons obtenu une unité répondant à l'expiration.

\section{$\left.2^{\circ}\right)$ Unités cardiaques.}

Pour 8 de ces unités la période de décharge se situait entre la fin du complexe $Q \mathrm{R} \mathrm{S}$ et le début de la phase $\mathrm{T}$ de l'électrocardiogramme. La neuvième pulsait à deux moments de l'activité cardiaque : avant et après le complexe $Q \mathrm{R} S$.

\section{0) Unités asophagiennes.}

Les récepteurs de l'œesophage ont été mis en jeu en gonflant un ballonnet placé dans la lumière de l'organe. Treize neurones ont répondu à la distension de l'œsophage thoracique et 2 à la distension de l'œesophage cervical. Ils étaient à adaptation lente, à 1'exception d'un seul qui s'adaptait rapidement.

\section{$\left.4^{\circ}\right)$ Unités provenant de l'estomac.}

Dans deux de nos expériences nous avons placé un ballonnet dans l'estomac. Nous avons alors détecté quatre unités à adaptation lente répondant à une distension comprise entre 70 et $100 \mathrm{~cm}^{3}$.

\section{DISCUSSION}

Nous pouvions craindre a priori de nous heurter à des difficultés venant d'une part de la dureté du ganglion, très riche en fibres conjonctives, d'autre part de l'amplitude des mouvements produits par la pulsation des carotides et des vaisseaux irriguant le ganglion. Ces inconvénients existent, mais sont loin de constituer des obstacles insurmontables.

Nous avons retrouvé divers résultats déjà obtenus à l'aide de la technique des fibres isolées. De plus nous avons pu mettre en évidence, dans l'œsophage thoracique et cervical, l'existence de plusieurs récepteurs à adaptation lente et d'un récep- 
teur à adaptation rapide. Il semble qu'une seule unité œsophagienne ait été étudiée auparavant (IGGO, I957).

La technique que nous avons utilisée nous paraît présenter les avantages suivants:

I) Il n'est pas nécessaire de sectionner le nerf plus ou moins complètement comme le font les auteurs qui utilisent la technique de la fibre isolée. Les fibres nerveuses restent ainsi en rapport avec leur centre trophique, qui a lui-même conservé son irrigation, et sont toutes en parfait état. L'organe dans lequel se trouvent les récepteurs a conservé la totalité de son innervation afférente et efférente.

2) L'enregistrement de l'activité unitaire des fibres amyéliniques est assez laborieux par la technique de la fibre isolée. Il semble par contre facile de recueillir le potentiel d'action du corps cellulaire.

3) S'il existe une somatotopie au niveau du ganglion plexiforme, ce que nous nous proposons de chercher, il sera sans doute possible d'étudier d'une manière prépondérante le type d'afférence souhaité (gastrique, œsophagien, etc.).

La dérivation de l'activité électrique des cellules sensitives en T à l'aide de microélectrodes, s'est donc révélée parfaitement applicable à l'étude des afférences intéroceptives. Elle nous semble constituer une technique de choix.

Rę̧u pour publication en décembre 1962.

\section{REMERCIEMENTS}

Travail exécuté avec l'aide d'une subvention de la Délégation générale à la Recherche scientifique et technique (Comité " Fonctions et Maladies du Cerveau ").

\section{SUMMARY}

SINGLE UNIT ACTIVITY OF AFFERENT VAGAL FIBRES RECORDED WITH MICROELECTRODES FROM THE LEVEL OF THE NODOSE GANGLION

The single unit activity of vagal sensory neurons has been recorded from the level of the nodose ganglion with extracellular microelectrodes. In 20 animals, we have been able to study r 30 units. Most of them were of respiratory origin, but we have also found units of cardiac, osophageal or gastric origin. It seems possible that the technique used should become the ordinary technique for the study of vegetative afferences.

\section{RÉFÉRENCES BIBL,IOGRAPHIQUES}

AdRian E. D., I933. Afferent impulses in the vagus and their effect on respiration. J. Physiol., Lonlon, 79, $33^{2}-35^{\circ}$.

IGgo A., I955. Tension receptors in the stomach and the urinary bladder. J.Physiol., London, 128, 593-607.

IGGO A., I957. Gastro intestinal tension receptors with unmyelinated afferent fibres in the vagus of the Cat. Quarl. J. exp. Physiol., 42, 130-143.

IGgo A., I958. The electrophysiological identification of single nerve fibres with particular reference to the slowest-conducting vagal afferent fibres in the Cat. J. Physiol., Lonton, 142, I Io- 26. 
PaINTAL A.S., 1952. Conduction velocity of single respiratory and cardiovascular afferent fibres in the cervical vagus. J. Physiol., London, $11 \%, 40 \mathrm{P}$.

PainTal A. S., I953. A study of right and left atrial receptors. J. Physiol, London, 120, 596-610.

Paintal A. S., I 954. A study of gastric stretch receptors. Their role in the peripheral mechanism of satiation of hunger and thirst. J. Physiol., London, 126, 255-270.

WhitTERIDGE D., I948. Afferent nerve fibres from the heart and lungs in the cervical vagus. J. Physiol., London, 107, 496-5I2. 